\title{
Melatonin Prevents Chronic Kidney Disease-Induced Hypertension in Young Rat Treated with Adenine: Implications of Gut Microbiota-Derived Metabolites
}

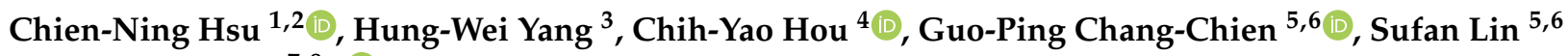 \\ and You-Lin Tain $7,8, * \mathbb{D}$
}

check for updates

Citation: Hsu, C.-N.; Yang, H.-W.; Hou, C.-Y.; Chang-Chien, G.-P.; Lin, S.; Tain, Y.-L. Melatonin Prevents Chronic Kidney Disease-Induced Hypertension in Young Rat Treated with Adenine: Implications of Gut Microbiota-Derived Metabolites. Antioxidants 2021, 10, 1211. https://doi.org/10.3390/ antiox10081211

\section{Academic Editors:}

Konrad Kleszczyński and Andrzej Slominski

Received: 29 June 2021

Accepted: 26 July 2021

Published: 28 July 2021

Publisher's Note: MDPI stays neutral with regard to jurisdictional claims in published maps and institutional affiliations.

Copyright: (c) 2021 by the authors. Licensee MDPI, Basel, Switzerland. This article is an open access article distributed under the terms and conditions of the Creative Commons Attribution (CC BY) license (https:/ / creativecommons.org/licenses/by/ $4.0 /)$.
1 Department of Pharmacy, Kaohsiung Chang Gung Memorial Hospital, Kaohsiung 833, Taiwan; cnhsu@cgmh.org.tw

2 School of Pharmacy, Kaohsiung Medical University, Kaohsiung 807, Taiwan

3 Institute of Medical Science and Technology, National Sun Yat-sen University, Kaohsiung 804, Taiwan; howardyang@imst.nsysu.edu.tw

4 Department of Seafood Science, National Kaohsiung University of Science and Technology, Kaohsiung 811, Taiwan; chihyaohou@webmail.nkmu.edu.tw

5 Center for Environmental Toxin and Emerging-Contaminant Research, Cheng Shiu University, Kaohsiung 833, Taiwan; guoping@csu.edu.tw (G.-P.C.-C.); linsufan2003@csu.edu.tw (S.L.)

6 Super Micro Mass Research and Technology Center, Cheng Shiu University, Kaohsiung 833, Taiwan

7 Department of Pediatrics, Kaohsiung Chang Gung Memorial Hospital and College of Medicine, Chang Gung University, Kaohsiung 833, Taiwan

8 Institute for Translational Research in Biomedicine, Kaohsiung Chang Gung Memorial Hospital and Chang Gung University, College of Medicine, Kaohsiung 833, Taiwan

* Correspondence: tainyl@cgmh.org.tw; Tel.: +886-975-056-995; Fax: +886-7733-8009

Abstract: Melatonin, a signaling hormone with pleiotropic biofunctions, has shown health benefits. Trimethylamine-N-oxide (TMAO) and asymmetric dimethylarginine (ADMA) are uremic toxins involved in the development of hypertension. TMAO originates from trimethylamine (TMA), a gut microbial product. ADMA is an endogenous nitric oxide (NO) synthase inhibitor. We examined whether melatonin therapy could prevent hypertension and kidney disease by mediating gut microbiota-derived metabolites and the NO pathway using an adenine-induced chronic kidney disease (CKD) young rat model. Six-week-old young Sprague Dawley rats of both sexes were fed a regular diet (C group), a diet supplemented with $0.5 \%$ adenine (CKD group), or adenine plus $0.01 \%$ melatonin in their drinking water (CKD + M group) for three weeks ( $N=8$ /group). Adenine-fed rats developed renal dysfunction, hypertension, renal hypertrophy and increased uremic toxin levels of TMAO and ADMA. Melatonin therapy prevented hypertension in both sexes and attenuated kidney injury in males. Melatonin reversed the changes to the plasma TMAO-to-TMA ratio induced by CKD in both sexes. Besides, the protective effects of melatonin were associated with restoration of gut microbiota alterations, including increased $\alpha$-diversity, and enhancement of the abundance of the phylum Proteobacteria and the genus Roseburia in male rats. Melatonin therapy also partially prevented the increases in ADMA in male CKD rats. Melatonin sex-specifically protected young rats against hypertension and kidney injury induced by CKD. The results of this study contribute toward a greater understanding of the interaction between melatonin, gut microbiota-derived metabolites, and the NO pathway that is behind CKD, which will help to prevent CKD-related disorders in children.

Keywords: asymmetric dimethylarginine; hypertension; chronic kidney disease; gut microbiota; melatonin; short-chain fatty acid; nitric oxide; trimethylamine-N-oxide; uremic toxin

\section{Introduction}

Around $10 \%$ of the global population has chronic kidney disease (CKD) [1]. Adult kidney disease can originate in early life [2]. Through early identification and prevention of 
CKD from adulthood to childhood, we have the potential to slow or reduce the progression of CKD in later life. Accordingly, World Kidney Day 2016 declared the need to focus on kidney disease in childhood or even at an earlier stage [3]. Thus far, several animal models have been used to study CKD. Among them, rats fed a diet containing adenine have gained attention for producing most of the features that mimic human CKD [4]. However, little information currently exists with regard to the use of adenine-induced CKD in young rats to investigate pediatric CKD [5].

The gut microbiome and its derived metabolites have been shown to play roles in mediating CKD [6]. In CKD, gut microbiota dysbiosis causes the accumulation of gutderived uremic toxins [7]. Trimethylamine-N-oxide (TMAO), a microbiota-derived uremic toxin, is related to increased cardiovascular events and mortality in the CKD population [8]. In the liver, TMAO originates from trimethylamine (TMA), a gut microbial product [9]. Prior research suggested that by increasing TMAO or TMA, high blood pressure (BP) could be induced in rats $[9,10]$. In a maternal adenine-induced CKD model, adult male offspring developed hypertension coinciding with abnormalities of the gut microbiota and a dysregulated TMA-TMAO pathway [11]. Besides, decreased short-chain fatty acid (SCFA) production has been related to increased BP [12].

Melatonin, N-acetyl-5-methoxytryptamine, is a biofunctional molecule widely distributed in nature [13]. Emerging evidence supports that early use of melatonin could be a potential strategy to prevent various chronic diseases in later life, including kidney disease [14]. It is known that melatonin, as well as its metabolites, act as antioxidants [15]. Nitric oxide (NO) deficiency is involved in the development of hypertension and kidney disease [16]. Asymmetric dimethylarginine (ADMA) can reduce NO production and induce oxidative stress [16]. In spontaneously hypertensive rats, melatonin has shown benefits against hypertension and oxidative stress via reduction of plasma ADMA and restoration of the NO pathway [17]. Although melatonin acts in a variety of ways to affect CKD [18], currently no information exists regarding its effect on gut microbiota-derived metabolites in pediatric CKD.

Accordingly, this research was carried out to elucidate whether melatonin therapy can protect young rats against CKD progression and hypertension and identify underlying protective mechanisms, focusing on the gut microbiota and its metabolites.

\section{Materials and Methods}

\subsection{Animal Studies}

All animal studies were approved by the Institutional Animal Ethics Committee (IACUC) of Chang Gung Memorial Hospital (permit number 2020031602). For the sample, 24 male and 24 female Sprague Dawley (SD) rats were purchased from BioLASCO Taiwan Co., Ltd. (Taipei, Taiwan). The rats were housed in an AAALAC-accredited facility. Food and water were available ad libitum. At six weeks of age, rats of both sexes received a regular diet (C group) or a diet supplemented with $0.5 \%$ adenine $(0.25 \mathrm{mg} / \mathrm{kg} /$ day $)$ for three weeks (CKD group). One group (CKD + M group) of the adenine-treated rats received $0.01 \%$ melatonin $(10 \mathrm{mg} / \mathrm{kg} /$ day, Sigma-Aldrich, St. Louis, MO, USA) in their drinking water simultaneously. The dose was selected based on our previous studies in rats $[19,20]$. We used the CODA noninvasive BP system (a tail-cuff method, Kent Scientific Corporation, Torrington, CT, USA) to determine the rats' BP at nine weeks old. Fresh fecal samples were collected, frozen, and stored at $-80^{\circ} \mathrm{C}$ until use. At nine weeks of age, the rats were anesthetized by intraperitoneally injecting ketamine $(50 \mathrm{mg} / \mathrm{kg}$ body weight) and xylazine $(10 \mathrm{mg} / \mathrm{kg}$ body weight) and were then euthanized by intraperitoneally injecting an overdose of pentobarbital for sacrifice. Heparinized blood samples were collected. The kidneys were subsequently collected. The plasma creatinine level was determined by high-performance liquid chromatography (HPLC) [12]. The animal care and experiments were conducted following established guidelines for the Care and Use of Laboratory Animals. 


\subsection{Analysis of TMA-TMAO Pathway}

The plasma levels of TMAO, TMA, and their dimethylamine (DMA) metabolites were analyzed by liquid chromatography-mass spectrometry (LC-MS) using previously described methods [21]. For the LC-MS analysis, an Agilent 6410 Series Triple Quadrupole mass spectrometer (Agilent Technologies, Wilmington, DE, USA) with an electrospray ionization source was employed. We used diethylamine as an internal standard. Using an Agilent Technologies 1200 HPLC system, chromatographic separation was carried out on a SeQuant ZIC-HILIC column $(150 \times 2.1 \mathrm{~mm}, 5 \mu \mathrm{m}$; Merck KGaA, Darmstadt, Germany $)$ protected by an Ascentis C18 column $(2 \mathrm{~cm} \times 4 \mathrm{~mm}, 5 \mu \mathrm{m}$; Merck KGaA). The eluate was monitored for DMA, TMA, and TMAO in multiple-reaction-monitoring mode using characteristic precursor-product ion transitions: $m / z 46.1 \rightarrow 30, m / z 60.1 \rightarrow 44.1$ and $m / z$ $76.1 \rightarrow 58.1$, respectively.

\subsection{Analysis of SCFAs}

Fecal concentrations of acetate, propionate, and butyrate were determined by gas chromatography-mass spectrometry (7890B, Agilent Technologies Wilmington, DE, USA) equipped with an automated sampler, as we previously published [21]. We used the standard analytical grades of butyrate (from Chem Service, West Chester, PA, USA), acetate, and propionate (Sigma-Aldrich) as internal standards. We used a DB-FFAP column ( $30 \mathrm{~cm} \times 0.25 \mathrm{~mm}, 0.25 \mu \mathrm{m}$; Agilent Technologies, Wilmington, DE, USA) for chromatographic separation. We used 2-ethylbutiric acid as the internal standard. An injection volume of $1 \mu \mathrm{L}$ with a split ratio of $5: 1$ was performed at $240{ }^{\circ} \mathrm{C}$. The fecal concentrations of SCFAs were adjusted by fecal weight and expressed as $\mathrm{mM} / \mathrm{g}$ feces.

\subsection{Gut Microbiota Compositions}

Stool samples were analyzed with metagenomics focused on the V3-V4 of the $16 \mathrm{~S}$ DNA gene using the methods published previously [21]. We used the Illumina MiSeq platform sequencing (Illumina, San Diego, CA, USA) and analyzed next-generation sequencing data at Biotools Co., Ltd. (Taipei, Taiwan). The sequences were clustered into operational taxonomic units (OTUs) using the USEARCH algorithm with a $97 \%$ sequence similarity threshold. Based on a representative sequence alignment with Fast-Tree, the phylogenetic relationships were constructed. We compared the diversity patterns of the microbial communities [22]. The alpha diversity was measured by Chao1 and the Shannon index [23]. We accessed the $\beta$-diversity of gut microbiota across groups using analysis of similarities (ANOSIM) and partial least squares discriminant analysis (PLS-DA) [24]. We used the linear discriminant analysis effect size (LEfSe) to discover high-dimensional biomarkers. The threshold of the logarithmic score (LDA) for discriminative features was set to four.

\subsection{Analysis of NO Pathway}

According to our validated protocol [21], high-performance liquid chromatography (HP series 1100; Agilent Technologies Inc., Santa Clara, CA, USA) with fluorescence detection of O-phthalaldehyde/3-mercaptopropionic acid (OPA/3MPA) derivatives was applied to determine the plasma levels of NO-related metabolites. These metabolites included Lcitrulline (the precursor of L-arginine), L-arginine (substrate for NO synthesis), ADMA, and symmetric dimethylarginine (SDMA; inhibitors of NO synthase). Homoarginine (Sigma) was used as the internal standard.

\subsection{Statistical Analysis}

We used the Statistical Package for the Social Sciences software (SPSS Inc., Chicago, IL, USA) for analysis. All data are expressed as the mean \pm the standard error of the mean (SEM). Comparisons within the three groups were analyzed by one-way analysis of variance (ANOVA) followed by Tukey's post hoc test. A $p$-value of less than 0.05 was regarded as statistically significant. 


\section{Results}

\subsection{Blood Pressure and Renal Function}

First, we observed that administration of adenine or melatonin did not induce death. The body weights (BWs) were lower in the CKD and CKD + M groups than the C group in both sexes (Table 1). In males, adenine administration caused a higher kidney weight and higher kidney weight-to-BW ratio in the CKD group vs. the C group, which was attenuated by melatonin therapy in males but not in females. At nine weeks of age, the systolic $\mathrm{BP}(\mathrm{SBP})$ and mean arterial pressure (MAP) were increased in the CKD group in males. Female rats receiving adenine also developed an increase in BP not only in SBP and MAP, but diastolic BP (DBP) as well, while the elevation of SBP in adenine-treated rats of both sexes was prevented by melatonin therapy. Additionally, Table 1 illustrates that adenine administration caused higher creatinine levels in the CKD groups of both sexes, which was partially prevented by melatonin therapy in males but not in females.

Table 1. Weights, blood pressure, and renal function.

\begin{tabular}{|c|c|c|c|}
\hline Groups & $\mathrm{C}$ & CKD & $\mathrm{CKD}+\mathrm{M}$ \\
\hline Male & $\mathrm{N}=8$ & $\mathrm{~N}=8$ & $\mathrm{~N}=8$ \\
\hline Body weight $(\mathrm{BW})(\mathrm{g})$ & $360 \pm 8$ & $261 \pm 11 *$ & $237 \pm 8 *$ \\
\hline Left kidney weight (g) & $1.78 \pm 0.07$ & $5.1 \pm 0.44$ * & $4.09 \pm 0.27 * \#$ \\
\hline Left kidney weight/100 g BW & $0.49 \pm 0.02$ & $1.94 \pm 0.12 *$ & $1.73 \pm 0.01 * \#$ \\
\hline Systolic blood pressure (mmHg) & $128 \pm 1$ & $144 \pm 1^{*}$ & $132 \pm 1 \#$ \\
\hline Diastolic blood pressure (mmHg) & $86 \pm 1$ & $87 \pm 1$ & $87 \pm 2$ \\
\hline Mean arterial pressure (mmHg) & $100 \pm 1$ & $106 \pm 1$ * & $102 \pm 1$ \\
\hline Creatinine $(\mu \mathrm{M})$ & $22.7 \pm 1$ & $322.7 \pm 25.7 *$ & $235.9 \pm 22.4 * \#$ \\
\hline Female & $\mathrm{N}=8$ & $\mathrm{~N}=8$ & $\mathrm{~N}=8$ \\
\hline Body weight (BW) (g) & $244 \pm 11$ & $187 \pm 6^{*}$ & $174 \pm 5 *$ \\
\hline Left kidney weight (g) & $1.12 \pm 0.03$ & $2.12 \pm 0.12 *$ & $1.91 \pm 0.13$ * \\
\hline Left kidney weight/100 g BW & $0.46 \pm 0.02$ & $1.14 \pm 0.05 *$ & $1.09 \pm 0.05 *$ \\
\hline Systolic blood pressure (mmHg) & $118 \pm 1$ & $132 \pm 1 *$ & $121 \pm 1 \#$ \\
\hline Diastolic blood pressure (mmHg) & $75 \pm 3$ & $84 \pm 3 *$ & $81 \pm 1 *$ \\
\hline Mean arterial pressure $(\mathrm{mmHg})$ & $89 \pm 2$ & $100 \pm 2 *$ & $94 \pm 1 \#$ \\
\hline Creatinine $(\mu \mathrm{M})$ & $23.4 \pm 1.3$ & $220.7 \pm 23.5^{*}$ & $190 \pm 18.2 *$ \\
\hline
\end{tabular}

\subsection{TMA-TMAO Pathway}

Table 2 shows the plasma levels of TMA, TMAO, and DMA. In males, melatonin therapy increased the plasma TMA level in the CKD + M groups compared to the other two groups (both $p<0.001$ ). The CKD and CKD + M groups had higher TMAO and DMA and a higher DMA-to-TMAO ratio in the plasma compared to the $C$ group. Adenine administration caused a higher TMAO-to-TMA ratio in the CKD group $(p<0.001)$, which melatonin therapy prevented $(p=0.001)$.

Similar to males, adenine-treated female rats had higher TMAO and DMA and a higher TMAO-to-TMA ratio vs. the controls. However, the increased plasma TMAO-toTMA ratio was prevented by melatonin therapy $(p<0.001)$. In addition, melatonin therapy caused the highest plasma DMA-to-TMAO ratio in the CKD + M group. 
Table 2. Plasma levels of TMA, TMAO and DMA.

\begin{tabular}{cccc}
\hline Groups & C & CKD & CKD + M \\
\hline Male & $\mathrm{N}=8$ & $\mathrm{~N}=8$ & $\mathrm{~N}=8$ \\
TMA (ng/mL) & $455 \pm 18$ & $466 \pm 26$ & $786 \pm 65^{*} \#$ \\
TMAO (ng/mL) & $301 \pm 18$ & $1427 \pm 92^{*}$ & $1376 \pm 164^{*}$ \\
DMA (ng/mL) & $111 \pm 5$ & $902 \pm 95^{*}$ & $809 \pm 84^{*}$ \\
TMAO-to-TMA ratio & $0.67 \pm 0.05$ & $3.13 \pm 0.27^{*}$ & $1.78 \pm 0.19^{*} \#$ \\
DMA-to-TMAO ratio & $0.38 \pm 0.02$ & $0.63 \pm 0.05^{*}$ & $0.62 \pm 0.07 *$ \\
Female & $\mathrm{N}=8$ & $\mathrm{~N}=8$ & $\mathrm{~N}=8$ \\
TMA $(\mathrm{ng} / \mathrm{mL})$ & $1653 \pm 291$ & $1877 \pm 253$ & $3543 \pm 185^{*} \#$ \\
TMAO $(\mathrm{ng} / \mathrm{mL})$ & $424 \pm 68$ & $2037 \pm 683^{*}$ & $1453 \pm 222^{*}$ \\
DMA $(\mathrm{ng} / \mathrm{mL})$ & $202 \pm 34$ & $755 \pm 51^{*}$ & $931 \pm 56^{*}$ \\
TMAO-to-TMA ratio & $0.3 \pm 0.05$ & $1.13 \pm 0.31 *$ & $0.41 \pm 0.06 \#$ \\
DMA-to-TMAO ratio & $0.47 \pm 0.02$ & $0.5 \pm 0.06$ & $0.7 \pm 0.07 *$ \\
\hline * & & &
\end{tabular}

${ }^{*} p<0.05$ vs. C; $\# p<0.05$ vs. CKD.

\subsection{Fecal SCFA Levels}

Table 3 illustrates that the changes in fecal SCFA concentrations were similar in both males and females. Adenine administration decreased the fecal concentrations of acetate, propionate, and butyrate in the $\mathrm{CKD}$ and $\mathrm{CKD}+\mathrm{M}$ groups compared to the $\mathrm{C}$ group.

Table 3. Fecal levels of acetate, propionate, and butyrate.

\begin{tabular}{cccc}
\hline Groups & C & CKD & CKD + M \\
\hline Male & $\mathrm{N}=8$ & $\mathrm{~N}=8$ & $\mathrm{~N}=8$ \\
Acetate, $\mathrm{mM} / \mathrm{gm}$ feces & $7.68 \pm 0.53$ & $5.49 \pm 0.6 *$ & $5.25 \pm 0.35 *$ \\
Propionate, $\mathrm{mM} /$ gm feces & $2.99 \pm 0.22$ & $1.45 \pm 0.44 *$ & $1.49 \pm 0.24 *$ \\
Butyrate, $\mathrm{mM} / \mathrm{gm}$ feces & $3.82 \pm 0.66$ & $1.15 \pm 0.24 *$ & $1.08 \pm 0.15 *$ \\
Female & $\mathrm{N}=8$ & $\mathrm{~N}=8$ & $\mathrm{~N}=8$ \\
Acetate, $\mathrm{mM} / \mathrm{gm}$ feces & $7.04 \pm 0.95$ & $3.93 \pm 0.46^{*}$ & $3.95 \pm 0.35 *$ \\
Propionate, $\mathrm{mM} / \mathrm{gm}$ feces & $2.73 \pm 0.35$ & $0.91 \pm 0.27 *$ & $0.54 \pm 0.09 *$ \\
Butyrate, $\mathrm{mM} / \mathrm{gm}$ feces & $5.1 \pm 0.71$ & $1.06 \pm 0.14 *$ & $0.7 \pm 0.09 *$ \\
\hline$p<0.05$ vs. C. & & &
\end{tabular}

\subsection{Gut Microbiota Compositions}

We investigated how adenine and melatonin affected the gut microbiota composition. As shown in Figure 1A, the male CKD group displayed a lower $\alpha$-diversity, represented by the Chao1 index, compared to the male C group $(p=0.033)$, which melatonin treatment prevented $(p=0.009)$. We next performed two $\beta$-diversity measures, the PLS-DA and ANOSIM, to compare the bacterial community similarity. Figure 1B illustrates scatterplots of the PLS-DA analysis with significant clustering according to the study group, showing that the gut microbiota structure of the C-group rats was distinctly altered by adenine and melatonin administration. The analysis of ANOSIM confirmed a significant difference in the gut microbiota among the three groups in males (all $p<0.05$ ), indicating that the three groups had distinct enterotypes.

At the phylum level, the major bacteria phyla found were Firmicutes, Bacteroidetes, Verrucomicrobia, Actinobacteria, and Proteobacteria in male rats (Figure 1C). The Firmicutes / Bacteroidetes (F/B) ratio, a microbial marker related to BP [25], did not differ among the three groups (Figure 1D). The abundance of the phylum Proteobacteria was greater in the CKD and CKD + M groups than that in the C group (Figure 1E). At the family level, CKD significantly increased the abundance of Deferribacteraceae compared to the control $(p=0.003)$, which was prevented by melatonin therapy $(p=0.002)$. 


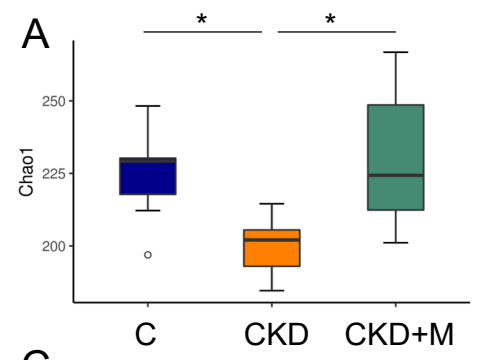

C

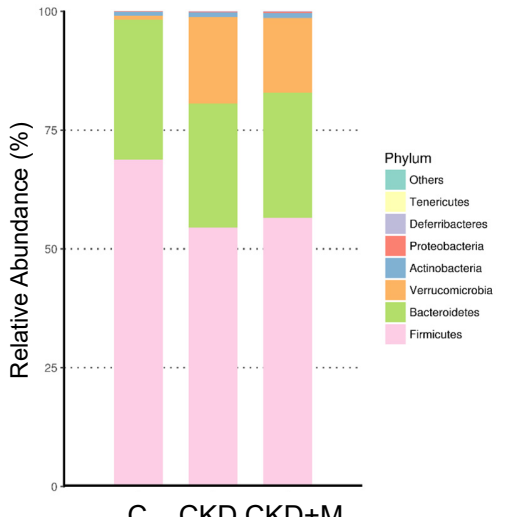

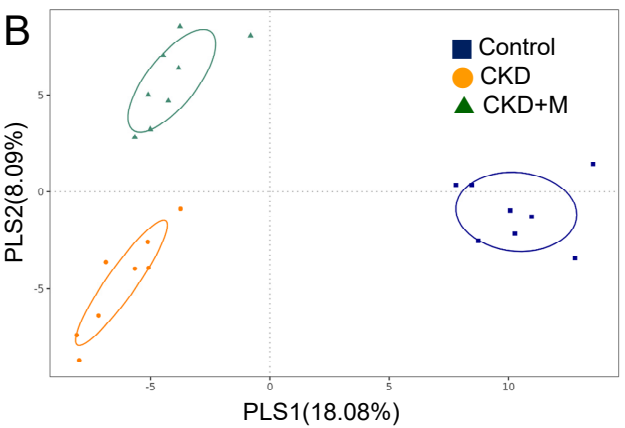

E
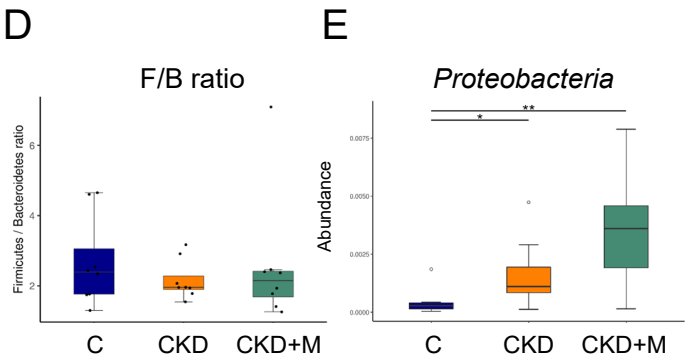

Figure 1. Gut microbiota composition in male rats. (A) $\alpha$-diversity represented by Chao1 analysis, (B) $\beta$-diversity measured by partial least squares discriminant analysis (PLS-DA), (C) relative abundance of the top phyla of the gut microbiota among the three groups, (D) the Firmicutes to Bacteroidetes ratio, and (E) the abundance of phylum Proteobacteria among the three groups. $\mathrm{N}=8$ /group. Data are shown as means $\pm \mathrm{SD}$. The single asterisk indicates $p<0.05$ vs. C. The double asterisk indicates $p<0.01$ vs. C.

Figure 2A illustrates the relative abundance of major genera in the three groups in males. At the genus level, CKD significantly increased the abundance of the genera Parabacteroides and Akkermansia compared to the controls. These increases were not affected by melatonin therapy in the CKD + M group vs. the CKD group (Figure $2 \mathrm{~B}, \mathrm{C}$ ). Conversely, CKD reduced the abundance of the genus Roseburia, which was prevented by melatonin therapy (Figure 2D). Additionally, the abundance of the genera Blautia, Eggerthella, and Erysipelatoclostridium were greater in the CKD and CKD + M groups vs. the control (Figure 2E-G).

Unlike males, the $\alpha$-diversity was not different among the three groups of females (Figure 3A). Figure 3B shows scatterplots of the PLS-DA analysis that are clearly separated, indicating the three groups displayed distinct enterotypes. Figure $3 \mathrm{C}$ shows the F/B ratio was higher in the female $\mathrm{CKD}$ and $\mathrm{CKD}+\mathrm{M}$ groups than that in the $\mathrm{C}$ group (Figure 3D). The female CKD + M group showed a greater abundance of the phylum Proteobacteria than the other two groups (Figure 3E).

In females, the abundance of the genera Parabacteroides, Akkermansia, Blautia, Eggerthella, and Erysipelatoclostridium were higher in the CKD and CKD + M groups than the $C$ group (Figure $4 \mathrm{~B}, \mathrm{C}, \mathrm{E}-\mathrm{G}$ ). However, the abundance of the genus Roseburia did not differ among the three groups (Figure 4D).

In Figure 5, the LEfSe algorithm identified several microbial markers that were significantly different between the groups. In the CKD group, the genera Akkermansia and Erysipelatoclostridium were in greater abundance in both sexes. Additionally, the genera Blautia in males and Parabacteroides in females were increased in the CKD group. Furthermore, the LEfSe analysis identified that melatonin significantly increased the abundance of the genus Parabacteroides in the male CKD + M group and the genera Blautia and Enterococcus in the female CKD + M group (Figure 5A,B). 

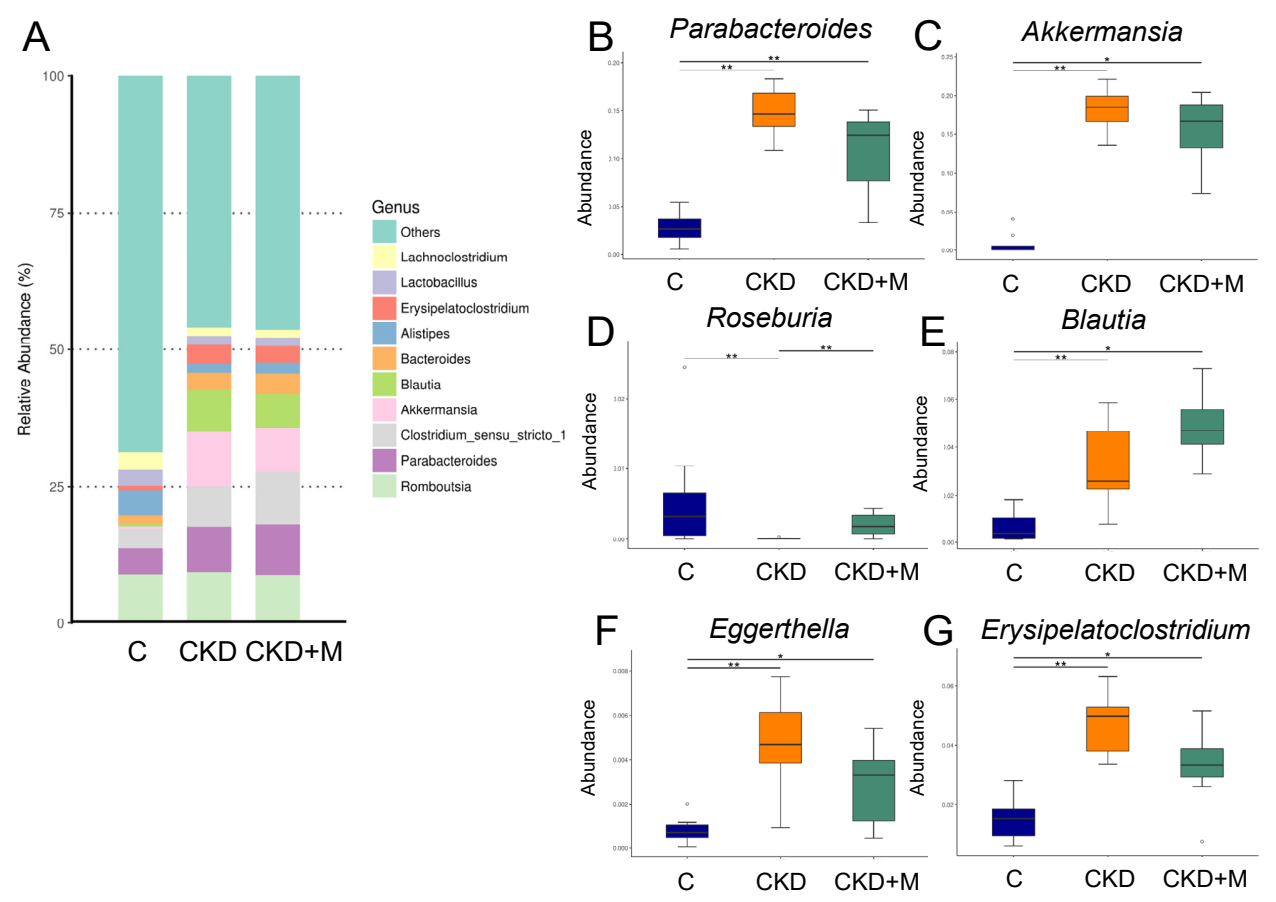

Figure 2. Gut microbiota at the genus level in male rats. (A) The relative abundance of the top 10 genera of the gut microbiota among the three groups. The abundance of genera (B) Parabacteroides, (C) Akkermansia, (D) Roseburia, (E) Blautia, (F) Eggerthella, and (G) Erysipelatoclostridium among the three groups. $\mathrm{N}=8$ /group. Data are shown as means $\pm \mathrm{SD}$. The single asterisk indicates $p<0.05$ vs. C. The double asterisk indicates $p<0.01$ vs. C.
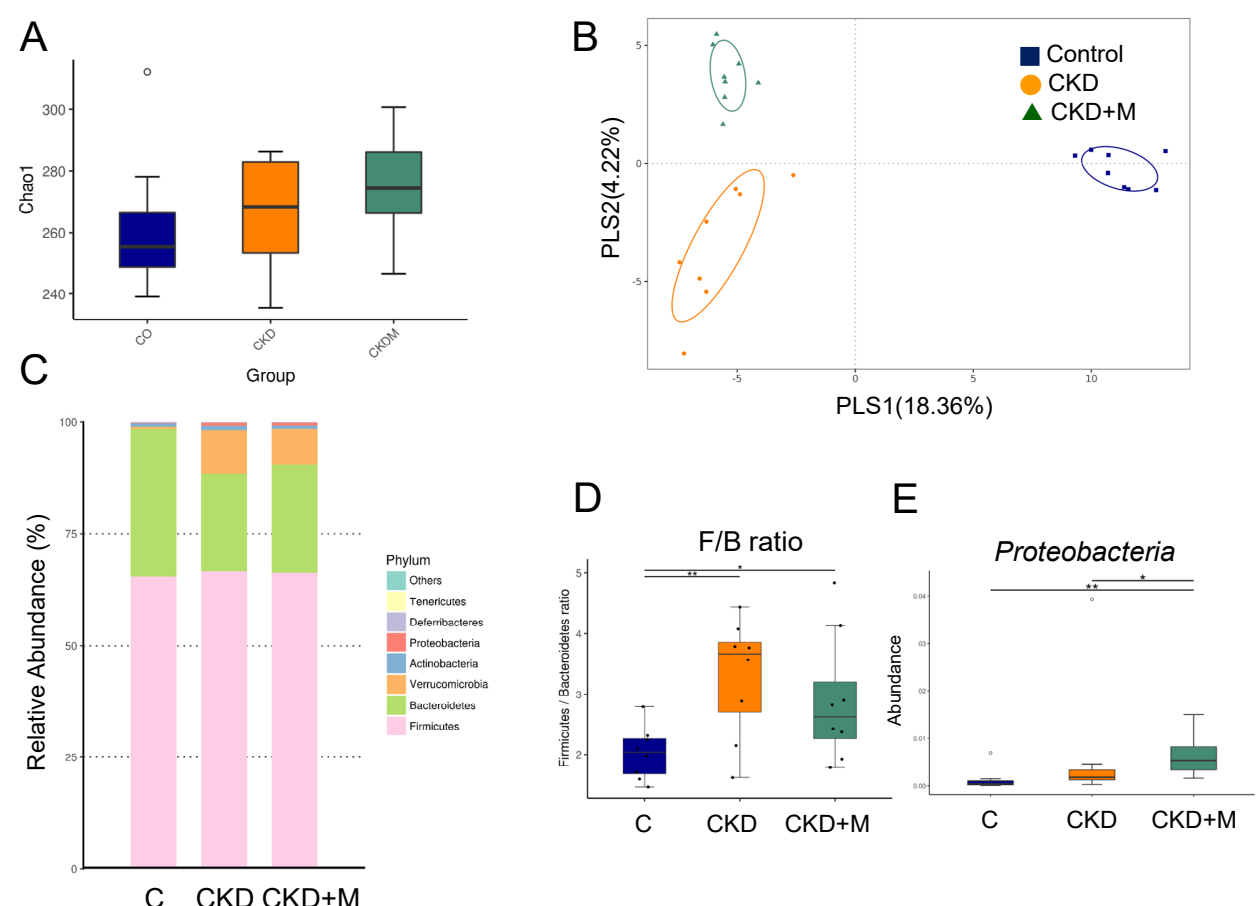

Figure 3. Gut microbiota composition in female rats. (A) $\alpha$-diversity represented by Chao1 analysis, (B) $\beta$-diversity measured by partial least squares discriminant analysis (PLS-DA), (C) relative abundance of the top phyla of the gut microbiota among the three groups, (D) the Firmicutes to Bacteroidetes ratio, and (E) the abundance of the phylum Proteobacteria among the three groups. $\mathrm{N}=8$ /group. Data are shown as means \pm SD. The single asterisk indicates $p<0.05$ vs. C. The double asterisk indicates $p<0.01$ vs. C. 

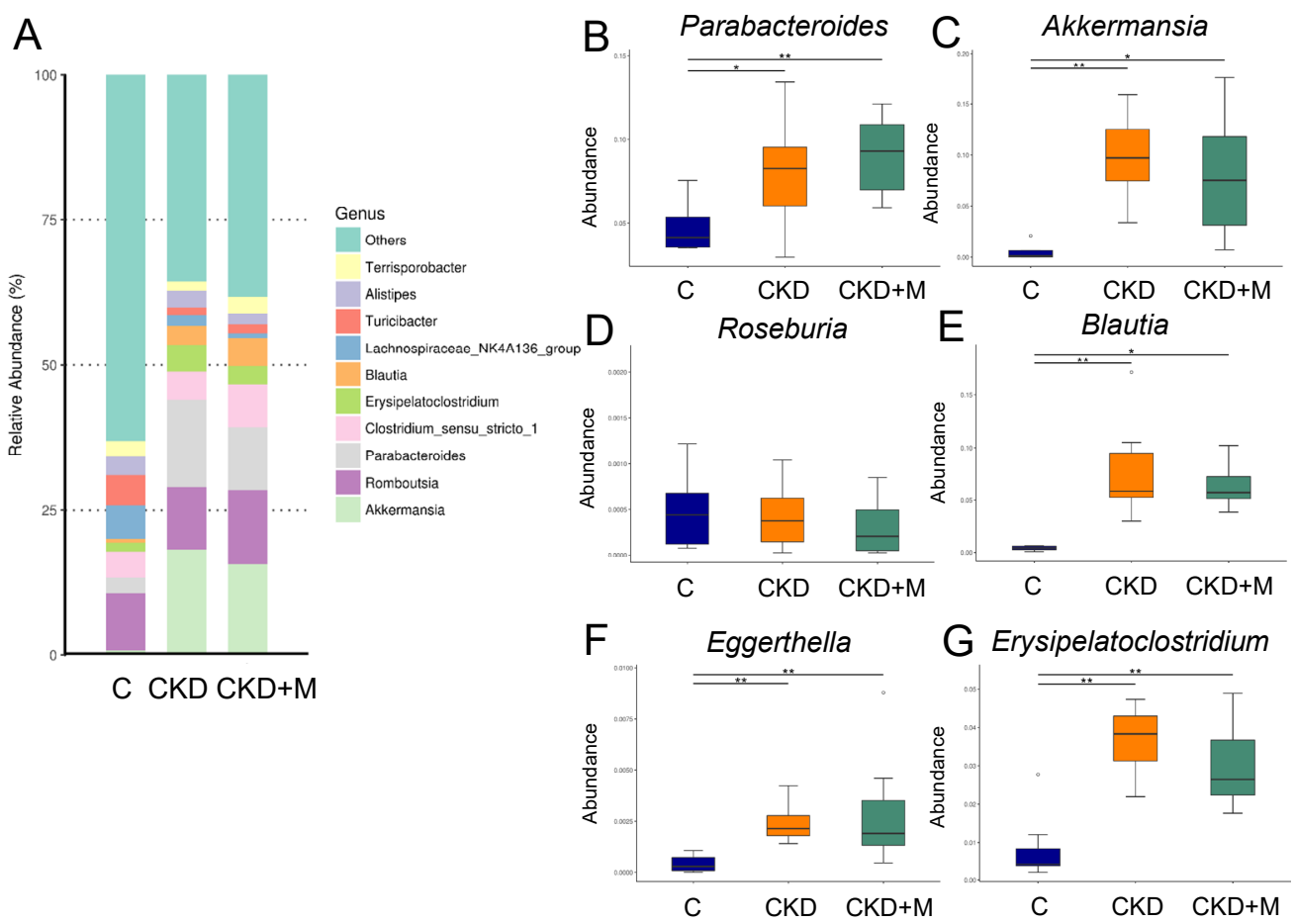

Figure 4. Gut microbiota at the genus level in female rats. (A) The relative abundance of the top 10 genera of the gut microbiota among the three groups. The abundance of genera (B) Parabacteroides, (C) Akkermansia, (D) Roseburia, (E) Blautia, (F) Eggerthella, and (G) Erysipelatoclostridium among the three groups. $\mathrm{N}=8$ /group. Data are shown as means $\pm \mathrm{SD}$. The single asterisk indicates $p<0.05$ vs. C. The double asterisk indicates $p<0.01$ vs. C.

A

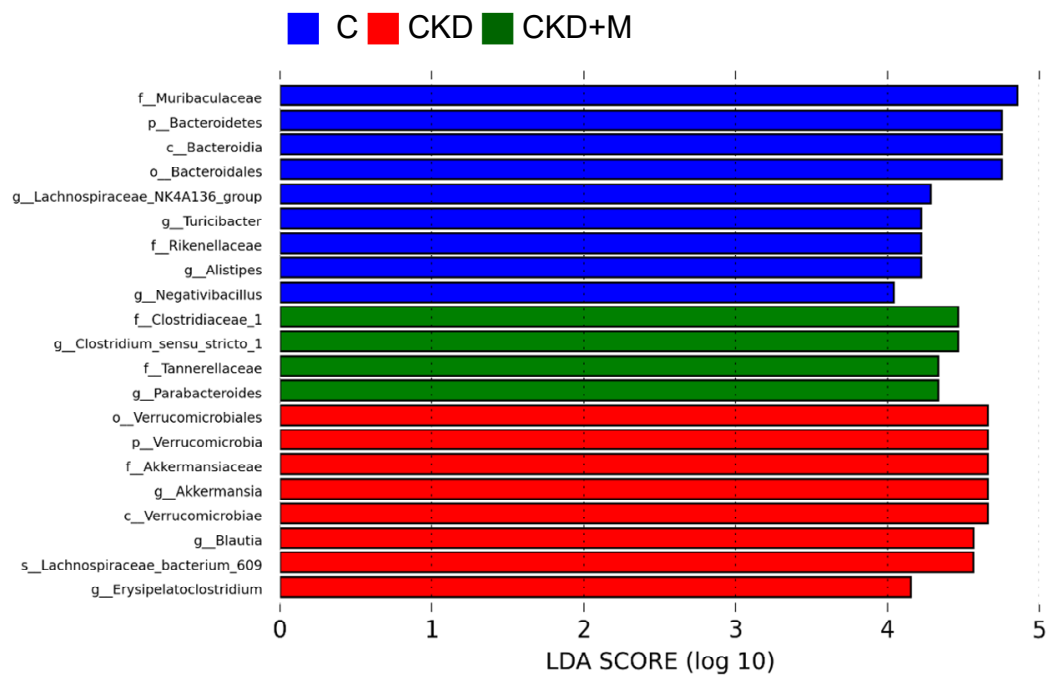

Figure 5. Cont. 
B

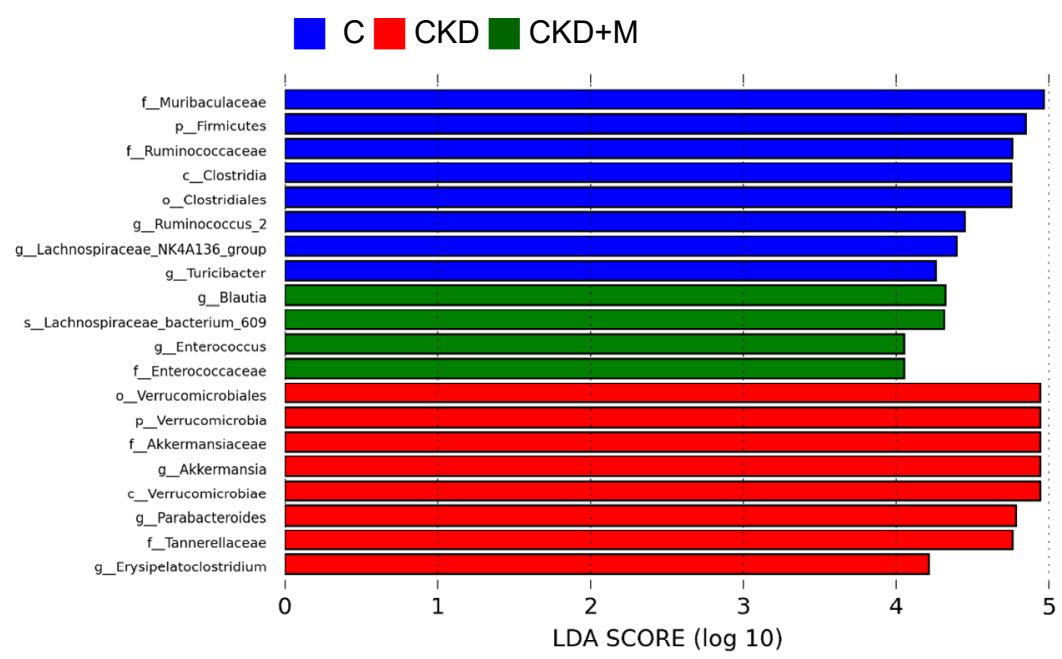

Figure 5. Linear discriminant analysis effect size (LEfSe) was applied to microbial marker discovery in metagenomic data. Here, are the most enriched and depleted bacterial taxa in (A) males and (B) females. C (blue) versus CKD (red) and CKD + M (green) are shown. Different taxonomic levels of bacteria are given, reaching from phylum down to the genus level. The threshold of the linear discriminant was set to four.

\subsection{NO-Related Parameters}

We next analyzed NO-related parameters as hypertension and CKD are closely linked to NO deficiency [16]. As shown in Table 4, there was no difference in the plasma Lcitrulline level among the three groups. The plasma ADMA level was higher, while the L-arginine-to-ADMA ratio was lower in the CKD group than the controls (both $p<0.05)$. In males, the plasma SDMA level was higher in the CKD and CKD + M groups vs. the controls. Melatonin therapy reduced the plasma L-arginine level and prevented increases in ADMA in CKD rats, while melatonin had a negligible effect on NO-related parameters in female CKD rats.

Table 4. Plasma levels of NO-related parameters.

\begin{tabular}{cccc}
\hline Groups & C & CKD & CKD + M \\
\hline Male & $\mathrm{N}=8$ & $\mathrm{~N}=8$ & $\mathrm{~N}=8$ \\
L-citrulline $(\mu \mathrm{M})$ & $53.7 \pm 1.6$ & $47.6 \pm 1.1$ & $46.7 \pm 1$ \\
L-arginine $(\mu \mathrm{M})$ & $165.8 \pm 2.8$ & $146.3 \pm 4$ & $118.8 \pm 1.4^{*}$ \\
ADMA $(\mu \mathrm{M})$ & $1.65 \pm 0.02$ & $2.4 \pm 0.04 *$ & $1.96 \pm 0.05 *$ \\
SDMA $(\mu \mathrm{M})$ & $1.21 \pm 0.01$ & $1.69 \pm 0.04^{*}$ & $1.61 \pm 0.02^{*}$ \\
Female & $102.4 \pm 2.9$ & $66 \pm 1.9 *$ & $58.8 \pm 1.6^{*}$ \\
L-arginine-to-ADMA ratio $(\mu \mathrm{M} / \mu \mathrm{M})$ & $\mathrm{N}=8$ & $\mathrm{~N}=8$ & $\mathrm{~N}=8$ \\
L-citrulline $(\mu \mathrm{M})$ & $56.1 \pm 0.9$ & $53.7 \pm 0.8$ & $55.8 \pm 1.2$ \\
L-arginine $(\mu \mathrm{M})$ & $166.4 \pm 2.5$ & $160.9 \pm 4.1$ & $140.4 \pm 3.2$ \\
ADMA $(\mu \mathrm{M})$ & $1.38 \pm 0.03$ & $2.1 \pm 0.07 *$ & $2.16 \pm 0.05^{*}$ \\
SDMA $(\mu \mathrm{M})$ & $1.58 \pm 0.07$ & $2.13 \pm 0.05$ & $1.72 \pm 0.02$ \\
L-arginine-to-ADMA ratio $(\mu \mathrm{M} / \mu \mathrm{M})$ & $123.1 \pm 3.4$ & $83.2 \pm 3.9 *$ & $65.8 \pm 1.2 *$ \\
\hline$p<0.05$ vs. C; $\# p<0.05$ vs. CKD. & & &
\end{tabular}

\section{Discussion}

The present study expands on earlier work regarding the health benefits of melatonin by showing that melatonin therapy prevents CKD-induced hypertension in both sexes and attenuates kidney injury in males; these results were achieved using an adenine-induced CKD model. We found it notable that the protective mechanisms of melatonin are related to the gut microbiota and TMA-TMAO pathway. 
Our study clearly shows that adenine, when administered at the same dose and for the duration, is more nephrotoxic in young rats than in adult rats [4,12]. Similar to adults, adenine-treated young rats developed common features of human CKD, such as renal dysfunction, renal hypertrophy, hypertension, and increased uremic toxin levels. These findings suggest that young rats are more vulnerable than adult rats to adenineinduced kidney injury, in support of this model used to study pediatric CKD. In line with previous studies showing melatonin can treat or prevent hypertension in various models of hypertension [26,27], this is the first report of melatonin therapy preventing CKD-induced hypertension in a young rat adenine-induced CKD model. Additionally, melatonin showed beneficial effects against kidney injury in a sex-dependent manner, with melatonin mainly attenuating kidney injury in males but not females. Although melatonin has been shown to interact with sex hormones reciprocally [28], little information currently exists with regard to sex differences in terms of the therapeutic response of melatonin in CKD. Sex differences exist in CKD and women seem to be somewhat protected from developing end-stage renal disease [29]. However, this is in contrast to our findings. Thus, more attention should be focused on the sex-specific effect of melatonin, particularly in other CKD models. Further studies on the sex differences in the response to melatonin therapy may support the discovery of a new tool for the prevention of CKD.

In support of prior research showing that gut microbiota dysbiosis contributes to kidney disease and hypertension $[6,12,30]$, adenine-induced CKD rats exhibited considerable alterations to the gut microbial composition. Consistent with previous studies reporting alterations of gut microbes in human and experimental CKD [25,30], young rats with CKD had a lesser abundance of beneficial microbes like Roseburia, but a greater abundance of the genera Parabacteroides and Akkermansia. Gut Roseburia spp. are part of the commensal bacteria with beneficial properties such as producing SCFAs, especially butyrate [31,32]. Our results also support a previous study showing that opportunistic pathogenic taxa like Parabacteroides were increased in a hypertensive gut microbiome, whereas SCFA producers such as Roseburia were decreased [33]. Moreover, consistent with our results, a previous study demonstrated that Akkermansia was increased, along with the progression of CKD [30]. Several studies have reported a potential association between a high $\mathrm{F} / \mathrm{B}$ ratio and hypertension $[11,25]$. However, our study failed to identify a reduction in the F/B ratio in the $\mathrm{CKD}+\mathrm{M}$ group, despite $\mathrm{BP}$ being reduced by melatonin therapy.

Consistent with previous studies demonstrating that uremic toxins are involved in CKD progression and hypertension [7-9], we observed that the elevated plasma level of TMAO coincided with hypertension and kidney dysfunction in adenine-induced young rats of both sexes with CKD. We observed that the plasma TMAO-to-TMA ratio was higher in the CKD group of both sexes, which melatonin prevented. Besides this, female CKD rats treated with melatonin had the highest plasma DMA-to-TMAO ratio among the three groups. The levels of plasma TMAO are determined by its renal excretion, its degradation to DMA, the TMA-to-TMAO conversion and TMA formation. A previous study showed a strong correlation between plasma TMAO levels and the glomerular filtration rate [34]. In CKD, increases in plasma TMAO can be mainly related to a decrease in the TMAO glomerular filtration. Besides this, TMAO can be metabolized to DMA or converted to TMA by TMAO reductase. Given that melatonin had a negligible effect on the renal function of female CKD rats, increased TMAO degradation, as reflected by the DMA-to-TMAO ratio, might be a protective mechanism behind CKD-induced hypertension. Another possible reason for increased TMAO might be increased TMAO-to-TMA conversion. Potentially, the TMAO-to-TMA ratio, as well as just the TMAO level, may be relevant to TMAO generation [35]. In accordance with the alteration to the TMA-TMAO pathway, alterations of the gut microbial community were observed. As Deferribacteraceae contributes to TMA production [36], similar to the TMAO-to-TMA ratio, male CKD rats had a higher abundance of Deferribacteraceae, which was prevented by melatonin therapy. Besides this, the phylum Proteobacteria was found to be the main contributor to the TMAO reductase pathway [37]. Melatonin therapy increased the TMA but decreased TMAO was associated with increases 
in the phylum Proteobacteria's abundance, which supports the notion that augmenting the TMAO reductase pathway provides a protective mechanism.

In this study, we also examined other metabolites derived from the gut microbiota, SCFAs, which have been proposed to act as a mechanism linking the gut microbiota to $\mathrm{BP}$ control [38]. Although our findings in conjunction with others suggest that reduction of SCFAs may underlie hypertension associated with CKD [39], the protective effects of melatonin against hypertension seem not to be related to SCFAs and their producing bacteria. Moreover, prior studies involving patients with hypertension and healthy controls showed that Blautia occurred at a lower abundance in the hypertension group than in the control group, whereas Eggerthella and Erysipelatoclostridium were present at higher levels $[40,41]$. These observations are in support of our findings showing a low abundance of Blautia and a high abundance of the genera Eggerthella and Erysipelatoclostridium in CKD rats presenting hypertension.

Another possible mechanism of melatonin protecting male rats against CKD-induced hypertension and kidney injury may be, at least in part, related to a reduction in ADMA. Like TMAO, ADMA is a known uremic toxin [42]. Melatonin has been reported to protect against hypertension or kidney injury, coinciding with a reduction in an oxidative stressinduced increase in ADMA [43,44]. Melatonin therapy lowered BP, attenuated kidney injury, and reduced ADMA concurrently in male CKD rats, which were in agreement with the findings of previous studies $[43,44]$.

Today, a broad spectrum of antihypertensive medication is accessible. However, the inadequate effectiveness and side effects of this medication lead patients with resistant hypertension to require invasive renal nerve denervation [45]. As a result, only a minority of these patients are satisfied with this therapy [45], not to mention its application in children. Thus, the search for a novel conservative treatment for resistant hypertension is unremitting. In children, CKD is the most common secondary cause of resistant hypertension. Melatonin is considered an essential candidate for the treatment of resistant hypertension [46] and our results show that melatonin even offers renoprotection beyond BP reduction, and therefore, has the potential future for clinical translation in the treatment of children with CKD.

Our study still has some limitations. One limitation is that we mainly analyzed gut microbiota and the NO pathway. Due to its pleiotropic biological activities, the protective effects of melatonin on hypertension and kidney injury might be attributed to other mechanisms. Another limitation is that we did not include a control $+\mathrm{M}$ group. The reason why we did not is that melatonin is currently used in humans and rats with a good safety profile [47]. However, it deserves further clarification as to whether the effects of melatonin therapy on young control rats differ from the effects on adult controls. Furthermore, we did not examine other dosages of melatonin; whether the physiological level of melatonin produces the same beneficial effects on CKD-induced hypertension awaits further evaluation. Last, the observations presented in our study are useful for indicating that melatonin has protective effects on CKD-induced hypertension by mediating gut microbiota-derived metabolites but are limited to testing in this model. Additional studies are required in other CKD models and in humans before melatonin can be translated into a clinical reality.

\section{Conclusions}

In this first adenine-induced pediatric CKD model, melatonin protected young rats from CKD-induced hypertension related to alterations of gut microbes involved in the TMA-TMAO pathway. The ADMA-NO pathway was also involved in CKD-induced hypertension. Providing a greater understanding of the alterations of gut microbiota and the derived metabolites that underlie CKD-induced hypertension, along with the beneficial effects of melatonin on protection from hypertension, our results can aid in preventing childhood hypertension and CKD progression. 
Author Contributions: Conceptualization, C.-N.H. and Y.-L.T.; data curation, C.-N.H., H.-W.Y., C.-Y.H., G.-P.C.-C., S.L. and Y.-L.T.; funding acquisition, Y.-L.T.; project administration, C.-N.H. and Y.-L.T.; writing - original draft, C.-N.H. and Y.-L.T.; writing—review and editing, C.-N.H., H.-W.Y., C.-Y.H., G.-P.C.-C., S.L. and Y.-L.T. All authors have read and agreed to the published version of the manuscript.

Funding: This research was funded by Chang Gung Memorial Hospital, Kaohsiung, Taiwan, grant No. CMRPG8K1011.

Institutional Review Board Statement: All animal studies were approved by the Institutional Animal Ethics Committee (IACUC) of Chang Gung Memorial Hospital (Permit Number 2020031602).

Informed Consent Statement: Not applicable.

Data Availability Statement: Data is contained within the article.

Conflicts of Interest: The authors declare no conflict of interest.

\section{References}

1. Luyckx, V.A.; Tonelli, M.; Stanifer, J.W. The global burden of kidney disease and the sustainable development goals. Bull. World Health Organ. 2018, 96, 414-422. [CrossRef]

2. Tain, Y.-L.; Hsu, C.-N. Developmental Origins of Chronic Kidney Disease: Should We Focus on Early Life? Int. J. Mol. Sci. 2017, 18, 381. [CrossRef]

3. Ingelfinger, J.R.; Kalantar-Zadeh, K.; Schaefer, F.; World Kidney Day Steering Committee. World Kidney Day 2016: Averting the legacy of kidney disease-focus on childhood. Pediatr. Nephrol. 2016, 31, 343-348. [CrossRef]

4. Diwan, V.; Brown, L.; Gobe, G.C. Adenine-induced chronic kidney disease in rats. Nephrology 2018, 23, 5-11. [CrossRef] [PubMed]

5. Claramunt, D.; Gil-Peña, H.; Fuente, R.; Hernández-Frías, O.; Santos, F. Animal models of pediatric chronic kidney disease. Is adenine intake an appropriate model? Nefrologia 2015, 35, 517-522. [CrossRef] [PubMed]

6. Ramezani, A.; Raj, D.S. The gut microbiome, kidney disease, and targeted interventions. J. Am. Soc. Nephrol. 2014, 25, 657-670. [CrossRef] [PubMed]

7. Graboski, A.L.; Redinbo, M.R. Gut-Derived Protein-Bound Uremic Toxins. Toxins 2020, 12, 590. [CrossRef]

8. Schiattarella, G.G.; Sannino, A.; Toscano, E.; Giugliano, G.; Gargiulo, G.; Franzone, A.; Trimarco, B.; Esposito, G.; Perrino, C. Gut microbe-generated metabolite trimethylamine-N-oxide as cardiovascular risk biomarker: A systematic review and dose-response meta-analysis. Eur. Heart J. 2017, 38, 2948-2956. [CrossRef]

9. Jaworska, K.; Hering, D.; Mosieniak, G.; Bielak-Zmijewska, A.; Pilz, M.; Konwerski, M.; Gasecka, A.; Kapłon-Cieślicka, A.; Filipiak, K.; Sikora, E.; et al. TMA, A Forgotten Uremic Toxin, but Not TMAO, Is Involved in Cardiovascular Pathology. Toxins 2019, 11, 490. [CrossRef]

10. Hsu, C.N.; Hou, C.Y.; Chang-Chien, G.P.; Lin, S.; Chan, J.Y.H.; Lee, C.T.; Tain, Y.L. Maternal resveratrol therapy protected adult rat offspring against hypertension programmed by combined exposures to asymmetric dimethylarginine and trimethylamine N-oxide. J. Nutr. Biochem. 2021, 93, 108630. [CrossRef]

11. Sun, S.; Lulla, A.; Sioda, M.; Winglee, K.; Wu, M.C.; Jacobs, D.R., Jr.; Shikany, J.M.; LloydJones, D.M.; Launer, L.J.; Fodor, A.A.; et al. Gut microbiota composition and blood pressure. Hypertension 2019, 73, 998-1006. [CrossRef] [PubMed]

12. Hsu, C.N.; Yang, H.W.; Hou, C.Y.; Chang-Chien, G.P.; Lin, S.; Tain, Y.L. Maternal Adenine-Induced Chronic Kidney Disease Programs Hypertension in Adult Male Rat Offspring: Implications of Nitric Oxide and Gut Microbiome Derived Metabolites. Int. J. Mol. Sci. 2020, 21, 7237. [CrossRef]

13. Hardeland, R.; Tan, D.X.; Reiter, R.J. Kynuramines, metabolites of melatonin and other indoles: The resurrection of an almost forgotten class of biogenic amines. J. Pineal Res. 2009, 47, 109-126. [CrossRef] [PubMed]

14. Tain, Y.L.; Huang, L.T.; Hsu, C.N. Developmental Programming of Adult Disease: Reprogramming by Melatonin? Int. J. Mol. Sci. 2017, 18, 426. [CrossRef] [PubMed]

15. Reiter, R.J.; Mayo, J.C.; Tan, D.X.; Sainz, R.M.; Alatorre-Jimenez, M.; Qin, L. Melatonin as an antioxidant: Under promises but over delivers. J. Pineal Res. 2016, 61, 253-278. [CrossRef] [PubMed]

16. Hsu, C.N.; Tain, Y.L. Regulation of Nitric Oxide Production in the Developmental Programming of Hypertension and Kidney Disease. Int. J. Mol. Sci. 2019, 20, 681. [CrossRef]

17. Tain, Y.L.; Huang, L.T.; Lin, I.C.; Lau, Y.T.; Lin, C.Y. Melatonin prevents hypertension and increased asymmetric dimethylarginine in young spontaneous hypertensive rats. J. Pineal Res. 2010, 49, 390-398. [CrossRef] [PubMed]

18. Hrenak, J.; Paulis, L.; Repova, K.; Aziriova, S.; Nagtegaal, E.J.; Reiter, R.J.; Simko, F. Melatonin and renal protection: Novel perspectives from animal experiments and human studies (review). Curr. Pharm. Des. 2015, 21, 936-949. [CrossRef]

19. Tain, Y.L.; Sheen, J.M.; Yu, H.R.; Chen, C.C.; Tiao, M.M.; Hsu, C.N.; Lin, Y.J.; Kuo, K.C.; Huang, L.T. Maternal Melatonin Therapy Rescues Prenatal Dexamethasone and Postnatal High-Fat Diet Induced Programmed Hypertension in Male Rat Offspring. Front. Physiol. 2015, 6, 377. [CrossRef] [PubMed] 
20. Tain, Y.L.; Lee, C.T.; Chan, J.Y.; Hsu, C.N. Maternal melatonin or N-acetylcysteine therapy regulates hydrogen sulfide-generating pathway and renal transcriptome to prevent prenatal N(G)-Nitro-L-arginine-methyl ester (L-NAME)-induced fetal programming of hypertension in adult male offspring. Am. J. Obstet. Gynecol. 2016, 215, 636. [CrossRef]

21. Hsu, C.N.; Chan, J.Y.H.; Wu, K.L.H.; Yu, H.R.; Lee, W.C.; Hou, C.Y.; Tain, Y.L. Altered Gut Microbiota and Its Metabolites in Hypertension of Developmental Origins: Exploring Differences between Fructose and Antibiotics Exposure. Int. J. Mol. Sci. 2021, 22, 2674. [CrossRef] [PubMed]

22. Morris, E.K.; Caruso, T.; Buscot, F.; Fischer, M.; Hancock, C.; Maier, T.S.; Meiners, T.; Müller, C.; Obermaier, E.; Prati, D.; et al. Choosing and using diversity indices: Insights for ecological applications from the German Biodiversity Exploratories. Ecol. Evol. 2014, 4, 3514-3524. [CrossRef] [PubMed]

23. Clarke, K.R.; Green, R.H. Statistical design and analysis for a 'biological effects' study. Mar. Ecol. Prog. Ser. 1988, 46, 213-226. [CrossRef]

24. Wagner, B.D.; Grunwald, G.K.; Zerbe, G.O.; Mikulich-Gilbertson, S.K.; Robertson, C.E.; Zemanick, E.T.; Harris, J.K. On the use of diversity measures in longitudinal sequencing studies of microbial communities. Front. Microbiol. 2018, 9, 1037. [CrossRef]

25. Yang, T.; Richards, E.M.; Pepine, C.J.; Raizada, M.K. The gut microbiota and the brain-gut-kidney axis in hypertension and chronic kidney disease. Nat. Rev. Nephrol. 2018, 14, 442-456. [CrossRef] [PubMed]

26. Paulis, L.; Simko, F. Blood pressure modulation and cardiovascular protection by melatonin: Potential mechanisms behind. Physiol. Res. 2007, 56, 671-684.

27. Baker, J.; Kimpinski, K. Role of melatonin in blood pressure regulation: An adjunct anti-hypertensive agent. Clin. Exp. Pharmacol. Physiol. 2018, 45, 755-766. [CrossRef] [PubMed]

28. Cipolla-Neto, J.; Amaral, F.G.; Soares, J.M., Jr.; Gallo, C.C.; Furtado, A.; Cavaco, J.E.; Gonçalves, I.; Santos, C.R.A.; Quintela, T. The crosstalk between melatonin and sex steroid hormones. Neuroendocrinology 2021. [CrossRef] [PubMed]

29. Iseki, K. Gender differences in chronic kidney disease. Kidney Int. 2008, 74, 415-417. [CrossRef]

30. Ren, Z.; Fan, Y.; Li, A.; Shen, Q.; Wu, J.; Ren, L.; Lu, H.; Ding, S.; Ren, H.; Liu, C.; et al. Alterations of the Human Gut Microbiome in Chronic Kidney Disease. Adv. Sci. 2020, 7, 2001936. [CrossRef]

31. Tamanai-Shacoori, Z.; Smida, I.; Bousarghin, L.; Loreal, O.; Meuric, V.; Fong, S.B.; Bonnaure-Mallet, M.; Jolivet-Gougeon, A. Roseburia spp.: A marker of health? Future Microbiol. 2017, 12, 157-170. [CrossRef] [PubMed]

32. Ducatelle, R.; Eeckhaut, V.; Haesebrouck, F.; Van Immerseel, F. A review on prebiotics and probiotics for the control of dysbiosis: Present status and future perspectives. Animal 2015, 9, 43-48. [CrossRef] [PubMed]

33. Yan, Q.; Gu, Y.; Li, X.; Yang, W.; Jia, L.; Chen, C.; Han, X.; Huang, Y.; Zhao, L.; Li, P.; et al. Alterations of the Gut Microbiome in Hypertension. Front. Cell Infect. Microbiol. 2017, 7, 381. [CrossRef]

34. Pelletier, C.C.; Croyal, M.; Ene, L.; Aguesse, A.; Billon-Crossouard, S.; Krempf, M.; Lemoine, S.; Guebre-Egziabher, F.; Juillard, L.; Soulage, C.O. Elevation of Trimethylamine-N-Oxide in Chronic Kidney Disease: Contribution of Decreased Glomerular Filtration Rate. Toxins 2019, 11, 635. [CrossRef]

35. Papandreou, C.; Moré, M.; Bellamine, A. Trimethylamine N-Oxide in Relation to Cardiometabolic Health-Cause or Effect? Nutrients 2020, 12, 1330. [CrossRef] [PubMed]

36. Koeth, R.A.; Wang, Z.; Levison, B.S.; Buffa, J.A.; Org, E.; Sheehy, B.T.; Britt, E.B.; Fu, X.; Wu, Y.; Li, L.; et al. Intestinal microbiota metabolism of L-carnitine, a nutrient in red meat, promotes atherosclerosis. Nat. Med. 2013, 19, 576-585. [CrossRef]

37. Jameson, E.; Doxey, A.C.; Airs, R.; Purdy, K.J.; Murrell, J.C.; Chen, Y. Metagenomic data-mining reveals contrasting microbial populations responsible for trimethylamine formation in human gut and marine ecosystems. Microb. Genom. 2016, 2, e000080. [CrossRef]

38. Pluznick, J.L. Microbial Short-Chain Fatty Acids and Blood Pressure Regulation. Curr. Hypertens. Rep. 2017, 19, 25. [CrossRef] [PubMed]

39. Felizardo, R.J.F.; Watanabe, I.K.M.; Dardi, P.; Rossoni, L.V.; Câmara, N.O.S. The interplay among gut microbiota, hypertension and kidney diseases: The role of short-chain fatty acids. Pharmacol. Res. 2019, 141, 366-377. [CrossRef]

40. Dan, X.; Mushi, Z.; Baili, W.; Han, L.; Enqi, W.; Huanhu, Z.; Shuchun, L. Differential Analysis of Hypertension-Associated Intestinal Microbiota. Int. J. Med. Sci. 2019, 16, 872-881. [CrossRef]

41. Palmu, J.; Salosensaari, A.; Havulinna, A.S.; Cheng, S.; Inouye, M.; Jain, M.; Salido, R.A.; Sanders, K.; Brennan, C.; Humphrey, G.C.; et al. Association Between the Gut Microbiota and Blood Pressure in a Population Cohort of 6953 Individuals. J. Am. Heart Assoc. 2020, 9, e016641. [CrossRef] [PubMed]

42. Tain, Y.L.; Hsu, C.N. Toxic Dimethylarginines: Asymmetric Dimethylarginine (ADMA) and Symmetric Dimethylarginine (SDMA). Toxins 2017, 9, 92. [CrossRef]

43. Tain, Y.L.; Huang, L.T.; Hsu, C.N.; Lee, C.T. Melatonin therapy prevents programmed hypertension and nitric oxide deficiency in offspring exposed to maternal caloric restriction. Oxid. Med. Cell Longev. 2014, 2014, 283180. [CrossRef] [PubMed]

44. Cheng, M.C.; Wu, T.H.; Huang, L.T.; Tain, Y.L. Renoprotective effects of melatonin in young spontaneously hypertensive rats with L-NAME. Pediatr. Neonatol. 2014, 55, 189-195. [CrossRef] [PubMed]

45. Hsu, C.N.; Huang, L.T.; Tain, Y.L. Perinatal Use of Melatonin for Offspring Health: Focus on Cardiovascular and Neurological Diseases. Int. J. Mol. Sci. 2019, 20, 5681. [CrossRef] [PubMed] 
46. Carey, R.M.; Calhoun, D.A.; Bakris, G.L.; Brook, R.D.; Daugherty, S.L.; Dennison-Himmelfarb, C.R.; Egan, B.M.; Flack, J.M.; Gidding, S.S.; Judd, E.; et al. Resistant Hypertension: Detection, Evaluation, and Management: A Scientific Statement from the American Heart Association. Hypertension 2018, 72, e53-e90. [CrossRef] [PubMed]

47. Simko, F.; Reiter, R.J.; Paulis, L. Melatonin as a rational alternative in the conservative treatment of resistant hypertension. Hypertens. Res. 2019, 42, 1828-1831. [CrossRef] 